\title{
Retrospective Study for Immune Thrombocytopenia Outcome in Children in Benha
}

\author{
A.A.Abouamer ${ }^{1}$, G.S.Abdel Motaleb ${ }^{1}$, K.A.Eid ${ }^{2}$, H.I.Abdelnaiem ${ }^{1}$ and A.M.Diab \\ ${ }^{1}$ Pediatric Dept., Faculty of Medicine, Benha Univ., Benha, Egypt \\ ${ }^{2}$ Pediatric Dept., Faculty of Medicine, Cairo Univ., Cairo, Egypt \\ E-Mail:heba1612003@yahoo.com
}

\begin{abstract}
Immune thrombocytopenia (ITP, formerly idiopathic thrombocytopenic purpura) is an acquired disease characterized by immune-mediated destruction of normal platelets and suppression of platelet production that is associated with variable bleeding symptoms. This review study is intended to dissect subtleties of safe thrombocytopenia (ITP) cases, assessment of treatment modalities and result among kids in Benha. Also, to decide the prognostic variables as rules for new cases at diagnosis. Techniques: One hundred and 50 instances of youngsters who were analyzed ITP and followed up in pediatric hematology department in Benha University Hospitals were incorporated. All the patients were exposed to Full history taking, Through clinical assessment, Laboratory examinations: Complete blood picture, Bone marrow sample (for indicated cases only) were done to affirm the diagnosis of ITP. Results : Of 150 patients, $79(52.7 \%)$ were guys, and $71(47.3 \%)$ were females, $59.5 \%$ of cases had intense ITP, $11 \%$ had persistent ITP, and $29.5 \%$ had ongoing ITP, we merged the first two groups. there was a critical contrast in platelet count between acute+persistent and ongoing ITP, the median platelet count was 12.4 x103 (range 2-54)in acute+persistent cases. We found that middle platelet count at finding was fundamentally lower for patients who showed acute+persistent ITP with middle platelet tally of 21.5 x103 (range 2-49) $(\mathrm{p}<0.001)$, We found young age had favorable outcome $(\mathrm{p}<0.001)$.
\end{abstract}

Keywords: Immune, Thrombocytopenia, ITP, Platelets.

\section{Introduction}

Immune thrombocytopenia (ITP, previously idiopathic thrombocytopenic purpura) is a procured infection portrayed by safe interceded devastation of typical platelets and concealment of platelet creation that is related with variable draining indications [1].

Essential ITP is characterized as a platelet check under $100 \times 109 / \mathrm{L}$ without different reasons for thrombocytopenia. The pinnacle occurrence is in youngsters 2 to $5 \mathrm{y}$ old enough. The yearly occurrence of ITP is somewhere in the range of 1.9 and 6.4 per 100,000 youngsters [2].

In pediatric patients, ITP influences guys all the more frequently in the more youthful years and females all the more regularly in the more seasoned years [3].

Patients with ITP present with disengaged thrombocytopenia (however weakness optional to blood misfortune might be available) and variable draining side effects which range from asymptomatic to serious, perilous dying. The determination of ITP is one of prohibition [4].

Youngsters who have no or mellow draining can be dealt with perception alone, paying little mind to platelet tally. In cases requiring treatment, intravenous immunoglobulin (IVIG), anti-D immunoglobulin, or corticosteroids can be regulated [5].

The recurrence of follow up blood tallies ought to be restricted to each 1 fourteen days at first and lesser from that point, to evade superfluous medical clinic visits and uneasiness [3].

When, regardless of rehashed dosages of first-line treatment,the sickness gets safe and ongoing enduring over a year, different therapies ought to be applied. Recently,the utilization of the thrombopoietin receptor agonist eltrombopag has been affirmed in kids more

established than one year with persistent ITP who have not reacted to the organization of first-line drugs [6].

In obstinate cases, different choices are the utilization of rituximab, a monoclonal enemy of CD20 fanciful neutralizer, or immunosuppressant drugs (e.g., cyclosporine, azathioprine, and mycophenolate mofetil. Hostile to D might be considered for first-line treatment in $\mathrm{Rh}+$ non splenectomized youngsters [3].

This review study is intended to dissect subtleties of insusceptible thrombocytopenia (ITP) cases, assessment of treatment modalities and result among kids in Benha. what's more, to decide the prognostic variables as rules for new cases at time of diagnosis.

\section{Patients and methods}

This is a retrospective study for analyze children with ITP less than18 years old who were diagnosed as primary immune thrombocytopenia who followed up at the pediatric hematology clinics over the course of 5 years.

\subsection{Inclusion criteria}

- Cases diagnosed with ITP.

- Children less than 18 years old

- Gender: both sexes.

\subsection{Exclusion criteria}

\section{Patient with other diseases}

Data of one hundred and fifty cases of children who were diagnosed as ITP and followed up in pediatric hematology clinics of Benha Hospitals were collected from files.

All the patients were subjected to Full history taking, Through clinical examination, Laboratory investigations: Complete blood picture, Bone marrow aspiration (for 
indicated cases only) were done to confirm the diagnosis of ITP.

\subsection{Ethical consideration}

\section{Confidential and anonymous data of patients}

The research ethics committee of benha faculty of medicine approved the study.

\subsection{Statistical analysis}

The collected data were tabulated and analyzed using SPSS version 16 soft ware (SpssInc, Chicago, ILL Company). Categorical data were presented as number and percentages, Chi square $\left(\chi^{2}\right)$ and Fisher's exact tests were used to analyze them. Quantitative data were tested for normality using Kolomogrov- Smirnov test, assuming normality at $\mathrm{P}>0.05$. Normally distributed variables were expressed as mean \pm standard deviation, while non parametric data were presented as median, IQR and range, and analyzed by Kruskal Wallis (KW) test.
Significant KW was followed by post hoc multiple comparisons using Bonferroni adjusted tests to detect the significant pairs. Matched non parametric variables were assessed by Friedman test. $\mathrm{P} \leq 0.05$ was considered significant.

\section{Results}

The age of the studied cases in the present study ranged between ( 1 year :18 years) with a median of age was 5.6 years old. , 79 were males, and 71 were females, sixty one percent lives in village and $39 \%$ lived in city, Our study showed that only $5.3 \%$ of the studied group had history of preceding vaccination, and about $83.3 \%$ of the studied group had no history of previous infection. Regarding diagnosis; $59.5 \%$ of cases had acute ITP , $11 \%$ of cases had persistent ITP and $29.5 \%$ of cases had chronic ITP. Fig 1.

About $70.8 \%$ of cases had skin bleeding in the form of petichie and echymosis Fig (2).

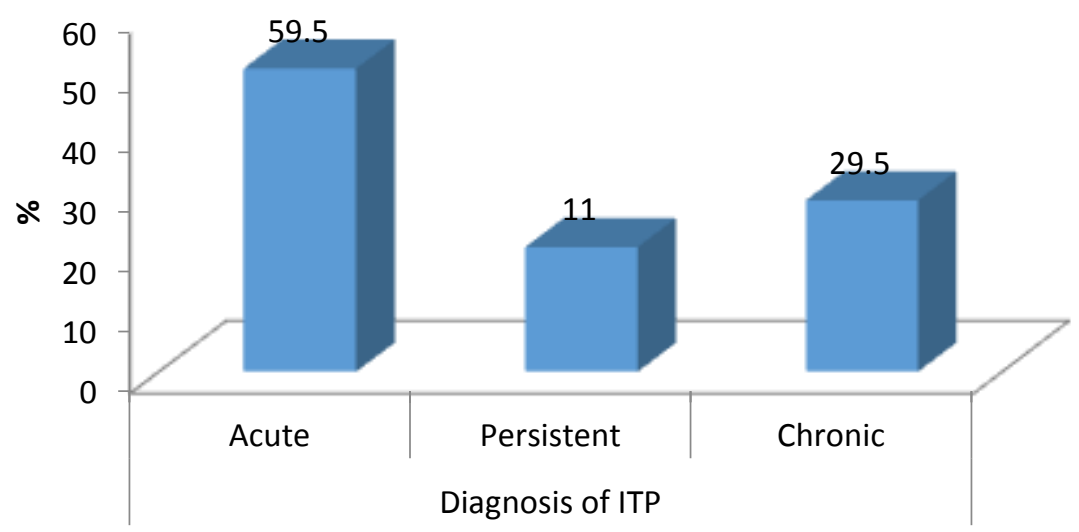

Fig (1) Diagnosis of the studied sample.

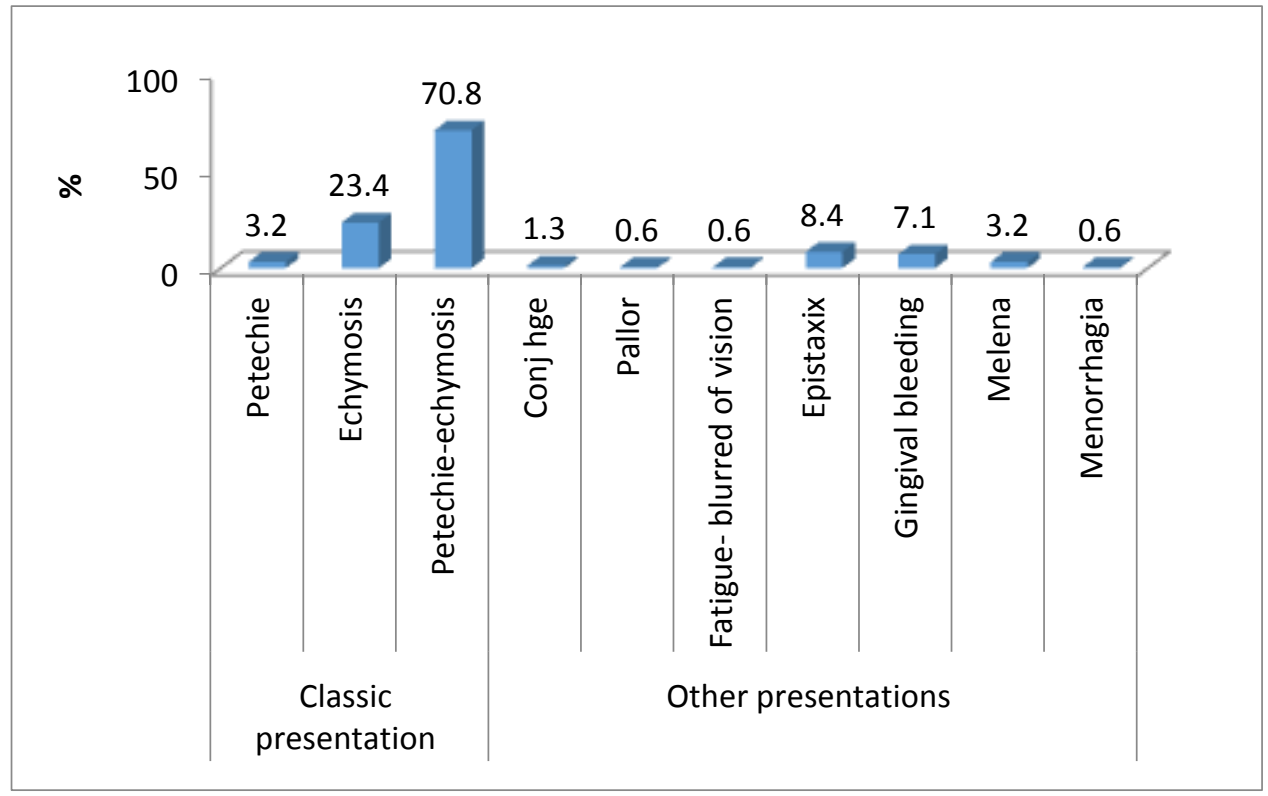

Fig (2) Type of bleeding among cases. 
Regarding age cases with younger age had favorable outcome as acute/persistent cases had mean age 4.8 years in comparison with mean of 7.7 years in ongoing cases $(\mathrm{p}<0.001)$. In present study $7.1 \%$ of patients had no medication, $92.9 \%$ received steroid therapy while $0.6 \%$ received IVIG and $29.9 \%$ received more than one drug Initial lines of treatment were steroids, intravenous immunoglobulins or no treatment and statistically there was no significant difference in outcome between initial lines of treatment $(\mathrm{p}=0.62)$. Our study showed that only $5.3 \%$ of the studied group had history of preceding vaccination, and about $83.3 \%$ of the studied group had no history of previous infection there was no effect of vaccine or infection on ITP $(p=0.06)$ Table (1).

Table (1) Vaccination among the studied sample.

\begin{tabular}{lccccc}
\hline & & \multicolumn{2}{c}{ Diagnosis } & \\
\hline & & & Acute/Persistent & Chronic & Total \\
\hline Vaccination & Yes & Count & 8 & 0 & 8 \\
& & $\%$ within Diagnosis & $7.5 \%$ & $.0 \%$ & $5.8 \%$ \\
& No & Count & 98 & 44 & 142 \\
Total & & $\%$ within Diagnosis & $92.5 \%$ & $100.0 \%$ & $94.2 \%$ \\
& & Count & 106 & 44 & 154 \\
& & $\%$ within Diagnosis & $100.0 \%$ & $100.0 \%$ & $100.0 \%$ \\
\hline
\end{tabular}

Our study showed a significant difference in platelet count between acute/persistent and chronic ITP, the median platelet count was $12.4 \times 10^{3}$ (range 2-54)in acute/persistent cases. We found that median platelet count at diagnosis was significantly lower for patients who developed acute/persistent ITP with median platelet count of $21.5 \times 10^{3}$ in chronic cases. (range 2-49) $(\mathrm{p}<0.001)$.

Table (2) Diagnosis according to platelets count over the first year.

\begin{tabular}{|c|c|c|c|c|c|c|c|c|}
\hline \multirow[t]{2}{*}{ PLTs $\left(\times 10^{3}\right)$} & \multicolumn{2}{|c|}{$\begin{array}{c}\text { Acute ITP } \\
(n=78)\end{array}$} & \multicolumn{2}{|c|}{$\begin{array}{c}\text { Persistent ITP } \\
(n=32)\end{array}$} & \multicolumn{2}{|c|}{$\begin{array}{c}\text { Chronic ITP } \\
(n=44)\end{array}$} & \multirow[t]{2}{*}{$\begin{array}{c}\text { KW } \\
\text { test }\end{array}$} & \multirow[t]{2}{*}{$\mathbf{P}$} \\
\hline & Median & Range & Median & Range & Median & Range & & \\
\hline At $2 w$ & $64 * \dagger$ & $7-509$ & 53.5 & $10-297$ & 36.0 & $11-966$ & 27.6 & $<0.001(\mathrm{HS})$ \\
\hline At $\mathbf{3 w}$ & $100.5^{* \dagger}$ & $20-780$ & 75.5 & $34-338$ & 78.5 & $24-674$ & 27.5 & $<0.001(\mathrm{HS})$ \\
\hline At $4 w$ & $111 * \dagger$ & $10-671$ & 86 & $27-304$ & 75.5 & $14-360$ & 44.9 & $<0.001(\mathrm{HS})$ \\
\hline At $1 \mathrm{~m}$ & $207.5 * \dagger$ & $69-762$ & 86.5 & $36-362$ & 85.5 & $15-500$ & 56.1 & $<0.001(\mathrm{HS})$ \\
\hline At $\mathbf{3 m}$ & $227.5^{*} \dagger$ & $114-472$ & 65.5 & $15-241$ & 56.5 & $15-312$ & 105.4 & $<0.001(\mathrm{HS})$ \\
\hline At $6 \mathrm{~m}$ & $289.5 * \dagger$ & $102-498$ & $92.0 *$ & $5-461$ & 52.5 & $3-98$ & 92.9 & $<0.001(\mathrm{HS})$ \\
\hline At $1 y$ & $\begin{array}{l}314 * \\
(\mathrm{n}=8)\end{array}$ & $254-415$ & $276.0 *$ & $119-465$ & 46.0 & $11-212$ & 61.9 & $<0.001(\mathrm{HS})$ \\
\hline
\end{tabular}

\section{$\dagger *$ significant in comparison with "Persistent" \\ *significant in comparison with "chronic"}

\section{Discussion}

Primary ITP is defined as a platelet count less than $100 \times 10^{3} / \mathrm{L}$ in the absence of additional causes of thrombocytopenia [7]. The peak incidence is in kids 2 to 5 years of age. The incidence of ITP is between 1.9 and 6.4 per 100,000 children annually [8].

Cases whom developed platelet count above $100 \times 10^{3}$ within first three months of the course of disease known as acute cases, those whom showed improvement after three months but before one year called persistent cases and those whom didn't develop improvement within first years and still having drop in the platelet count after one year called chronic cases [9].

In this study, $59.5 \%$ of cases had intense ITP, $11 \%$ had tireless ITP, and $29.5 \%$ had ongoing ITP. G.R
Buchanan [9] additionally found that 27\% of cases created constant ITP. Singh et al., [10] found that ongoing ITP addresses $15-20 \%$ of cases.

There was no genuinely critical distinction between sex and the course of the infection this is predictable with Gungor et al., [11]. As to conveyance, our examination uncovered that females had same frequency to be constant as guys. This is not steady with Chapin $\mathbf{J}$ et al., [12], who discovered female is a marker for chronicity; this might be clarified by that sex chemicals, as in other invulnerable problems, may assume a part in the weakness to chronicity. Notwithstanding affecting the safe framework, sex chemicals may likewise adjust the clinical picture and reaction to treatment. In the current examination, the residency of patients was not 
essentially altering the course of the illness. Likewise, there was no critical connection between's the season wherein patients demonstrated their first manifestations and the sickness' course; this is upheld by Chaplin [13]. Likewise, the kind of bleeding didn't essentially adjust the illness' course; this outcome was upheld by Chaplin [13].

Our study showeded that bleeding of intense instances of ITP $70.8 \%$ patients had skin bleed, $23.5 \%$ got echymosis while $3.2 \%$ petechie, This concur with Cines et al., [14], who demonstrated that most of intense instances of ITP were presented with skin symptoms in the clinic, (96\%) had at any rate at least one skin symptom. Of the individuals who got admitted (60\%) were with echymosis, only 6 patients $(24 \%)$ were having petechie, and (12\%) got the two forms. These outcomes additionally can't help contradicting cole et al., [15], as they indicated that kids with the intense sort of the illness show bleeding via mucus membrane more, There was no effect of previous infection on sort of illness this is against Khan et al.,[16] who found that previous infection had gotten all the more frequently bleeding $(78.3 \%)$.

In persistent cases in our investigation $70.4 \%$ got Azathioprine, $6.8 \%$ got Eltrombobag ,18.1\% got both Azathioprine and Eltrombobag and 2.3\% got both Azathioprine and cyclosporine, there was no measurably critical contrast between the line of treatment and the course of the illness.

In our investigation about $70 \%$ of patients got Azathioprine indicated in general reaction. This outcome is reliable with Poudyal et al. [17] who announced reaction to Azathioprine in $75 \%$ of cases .

Around $86 \%$ of patients getting Eltrombopag achieved demonstrated total reaction during treatment with eltrombobag .This outcome is steady with González-López et al. [18] who detailed reaction to Eltrombopag in $89 \%$ of cases.

Regarding vaccination no any effect in the type of ITP this consistent with George A.F [19].

\section{Conclusion}

Younger age and low platelet count at diagnosis had favorable outcome.

\section{References}

[1] K. M. Haley, "Background of Immune Thrombocytopenia," in Immune Hematology, Springer; Vol. 2018(1), PP.3-15, 2018.

[2] D. R. Terrell, L. A. Beebe, S. K. Vesely, B. R. Neas, J. B. Segal, J. N. George, "The incidence of immune thrombocytopenic purpura in children and adults: a critical review of published reports," Am. J. Hematol; Vol. 85(3), PP.174-180, 2010.

[3] C. E. Neunert ,"Bleeding manifestations and management of children with persistent and chronic immune thrombocytopenia: data from the Intercontinental Cooperative ITP Study Group (ICIS)," Blood, J. Am. Soc. Hematol; Vol. 121(22), PP.4457-4462, 2013.
[4] N. Cooper, "State of the art-how I manage immune thrombocytopenia," Br. J. Haematol; Vol. 177(1), PP.39-54, 2017.

[5] D. Provan "'International consensus report on the investigation and management of primary immune thrombocytopenia," Blood; Vol. 115(2), PP.168$186,2010$.

[6] C. B. Burness, G. M. Keating, K. P. Garnock-Jones, "Eltrombopag: a review in paediatric chronic immune thrombocytopenia," Drugs; Vol. 76(8), PP.869-878, 2016.

[7] C Neunert , W Lim , MCrowther, et al.,:The American Society of Hematology evidence-based practice guideline for immune thrombocytopenia. Blood 2011; 117:4190, 2011.

[8] DR Terrell, LA Beebe, SK Vesely :The incidence of immune thrombocytopenic purpura in children and adults: a critical review of published reports. Amer J Hematol. ;85:174-80,2010.

[9] G.R Buchanan: Immune Thrombocytopenia during Childhood: New Approaches to Classification and Management. The J., Pediatrics. 165 (3) 437-439, (2014).

[10] G. Singh, D. Bansal, N. A. M. Wright, "Immune thrombocytopenia in children: Consensus and controversies," Indian J. Pediatr; Vol. 87(2), PP.150-157, 2020.

[11]T. Güngör , "Retrospective evaluation of children with immune thrombocytopenic purpura and factors contributing to chronicity," Pediatr. Neonatol; Vol. 60(4), PP.411-416, 2019.

[12] J Chapin, C S Lee, H Zhang, et al.: Gender and duration of disease differentiate responses to rituximab-dexamethasone therapy in adults with immune thrombocytopenia. American J., Hematology; 91: 907-911, (2016).

[13] D.D Chaplin.:Overview of the immune response. J. Allergy Clin. Immunol. 125:S3-23, 2010.

[14]D.B Cines., A Cuker. and J.W Semple: Pathogenesis of immune thrombocytopenia. Presse Med.43:e49-e5, 2014.

[15] C Cole .Lessons in the diagnosis and management of immune thrombocytopenic purpura in children. J., Paediatrics and Child Health; 53: 833-835

[16] B. S. Poudyal, B. Sapkota, G. S. Shrestha, S. Thapalia, B. Gyawali, S. Tuladhar, "Safety and efficacy of azathioprine as a second line therapy for primary immune thrombocytopenic purpura," $J$. Nepal Med. Assoc; Vol. 55(203), PP.16-21, 2017.

[17]M.A Khan, H Mydra and A Nevarez : Clinical Practice Updates in the Management of Immune Thrombocytopenia $\mathrm{p}$ and $\mathrm{T}$ Vol. 42 no.12 756763,2017.

[18] T. J. González-López , "Successful discontinuation of eltrombopag after complete remission in patients with primary immune thrombocytopenia," Am. J. Hematol; Vol. 90(3), PP.40-43, 2015.

[19] A.F George (2015): Platelet structure and function . American Society for Clinical Laboratory Science. 28 (2) 125-131. 